\title{
Primary Cutaneous Adenoid Cystic Carcinoma Arising in Umbilicus
}

\author{
Seok Joo Lee · Woo Ick Yang · Sang Kyum Kim \\ Department of Pathology, Yonsei University College of Medicine, Seoul, Korea
}

Primary cutaneous adenoid cystic carcinoma (PCACC) is a rare adnexal tumor, first described by Boggio in 1975. ${ }^{1}$ Fewer than 70 cases have been reported in the English literature. It has an indolent course and a high tendency to recur but manifests low potential for distant metastases.

Herein, we report a case of 66-year-old male who presented with a slow-growing erythematous lesion in the umbilicus. Upon histopathological examination, the tumor exhibited features of PCACC. The patient was treated with Mohs micrographic surgery. To our knowledge, this is the first reported case of a PCACC in the umbilicus. We discuss the clinicopathological characteristics of PCACC and include review of the literature.

\section{CASE REPORT}

A 66-year-old man with a medical history of congestive heart failure presented with a single, ill-defined, bean-sized erythematous lesion with tenderness in the umbilicus (Fig. 1A). Because the lesion had been nearly asymptomatic, except for mild tenderness, the patient had not sought a medical treatment. He eventually became concerned about the abnormal appearance of it and visited an outpatient clinic. An incisional biopsy was done and the patient was referred to our institution. Mohs micrographic surgery was performed. The cut surface of the specimen revealed a firm infiltrative mass involving dermis and subcutis (Fig. 1B). Hematoxylin and eosin stained slides were reviewed by three pathologists (S.K.K., S.J.L., and W.I.Y.). Microscopic examination revealed infiltrative nests and cords of basaloid tu-

\section{Corresponding Author}

Sang Kyum Kim, MD, PhD

Department of Pathology, Yonsei University College of Medicine, 50-1 Yonsei-ro Seodaemun-gu, Seoul 03722, Korea

Tel: +82-2-2228-6751, Fax: +82-2-2227-7939, E-mail: nicekyumi@yuhs.ac

Received: October 6, 2015 Revised: November 18, 2015

Accepted: November 23, 2015 mor cells with cribriform or tubular architecture in the dermis and subcutaneous tissue without connection to the epidermis (Fig. 2A). Abundant mucin was present and perineural invasion by the tumor cells was often observed (Fig. 2B, C). Based upon these histologic findings, primary or metastatic adenoid cystic carcinoma (ACC), adenoid cystic variant of basal cell carcinoma, and microcystic adnexal carcinoma entered in the differential diagnoses. Immunohistochemistry with antibodies against type III receptor tyrosine kinase KIT (CD117, catalog \#A4502, Dako, Carpinteria, CA, USA) and epithelial membrane antigen (EMA; catalog \#M0613, Dako) was performed with an automated immunohistochemical staining instrument (VentanaDiscovery XT, Ventana Medical System Inc., Oro Valley, AZ, USA). The tumor cells showed strong expressions of EMA (Fig. 3A) and KIT (Fig. 3B). The histological findings and immunohistochemical results indicated that the tumor was ACC. There was no other palpable mass on physical examination and there was no evidence of metastasis in the imaging study. Therefore, the tumor was diagnosed as a PCACC. The patient was discharged without complication. At postoperative follow-up after 6 months, he was healthy without evidence of local recurrence.

This retrospective study was approved by the Institutional Review Board of Yonsei University Medical Center (approval No. 4-2015-0695).

\section{DISCUSSION}

ACC is a rare type of cancer that usually occurs in the head and neck, particularly in the salivary glands. However, it may develop in any site where secretory glands are present. It has been reported in the breast, lacrimal gland, lung, brain, uterine cervix, trachea, paranasal sinus, and skin. ${ }^{1}$ The etiology of PCACC is not entirely clear. However, the World Health Organization (WHO) recently concurred with the prevailing view that the 
tumor originates from the apocrine glands. In the WHO Classifuation of Skin Tumors, ACC is classified as an adnexal neoplasm. ${ }^{2}$

Clinically, the tumor is a firm, poorly circumscribed, slowly growing nodule that is flesh-colored or red, and may or may not be accompanied by pain and tenderness. ${ }^{3}$ Tumors that occur on the head and neck represent $46 \%$ of PCACCs and many of these are on the scalp. Of the remaining tumors that have been reported, $15 \%$ occur on the trunk, $17 \%$ on the upper limbs or in the area of the pectoral girdle, and $13 \%$ on the lower limbs or in the area of the pelvic girdle. ${ }^{4}$ To our knowledge, this is the first reported case of a PCACC in the umbilicus.

For the diagnosis of PCACC, it is essential to rule out the possibility that the tumor has metastasized from elsewhere, particularly from the salivary glands. ACC of the salivary glands is aggressive and highly metastatic, with a high rate of local recurrence, while PCACC rarely metastasizes: metastases of PCACC

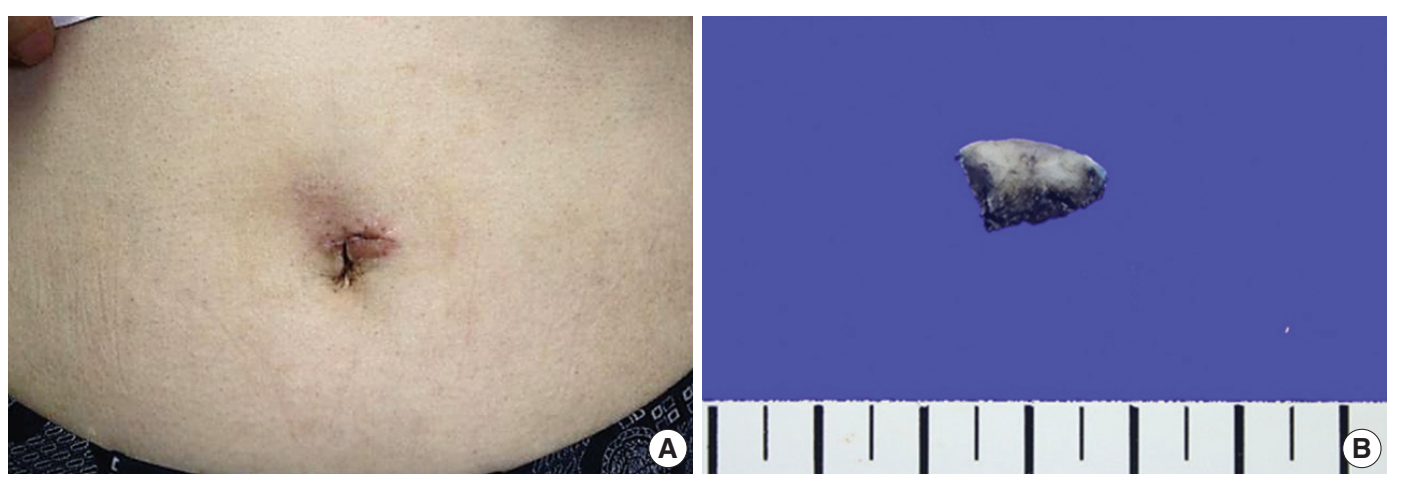

Fig. 1. Macroscopic findings of tumor arising in umbilicus. (A) An ill-defined bean-sized erythematous lesion on the umbilicus. (B) In sections of the specimen from the Mohs micrographic surgery, cut surfaces had an infiltrative firm mass.
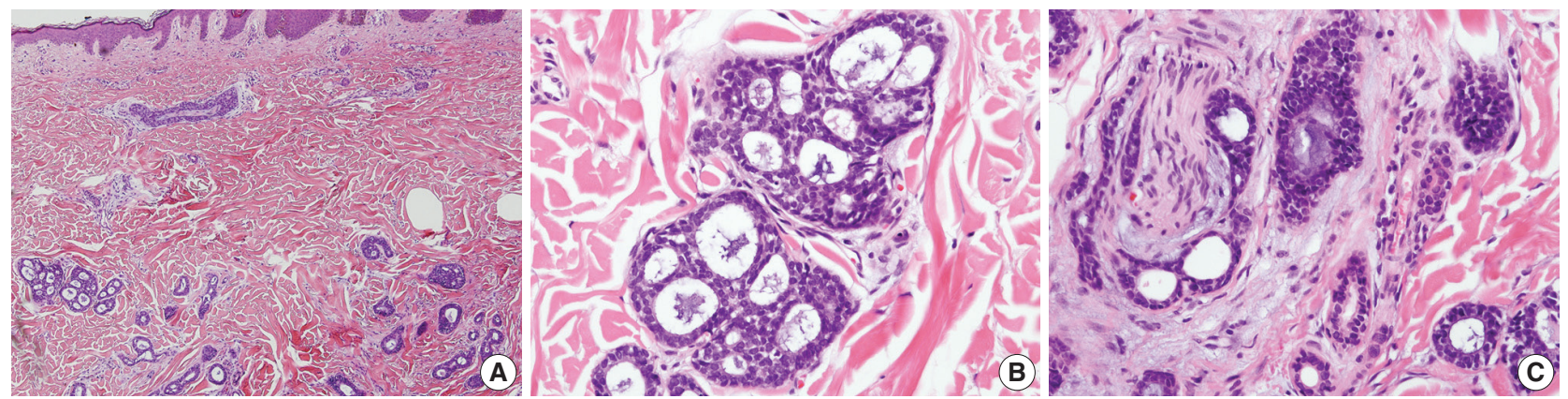

Fig. 2. Microscopic findings of primary cutaneous adenoid cystic carcinoma. (A) Images of basaloid cells that have infiltrated the dermis and the subcutaneous layer, but not the epidermis. Note the tumor cells' arrangement in nests and their cribriform pattern. (B) Images showing the cribriform and tubular growth patterns of the tumor, which also have a solid portion. (C) Perineural invasion by the tumor.
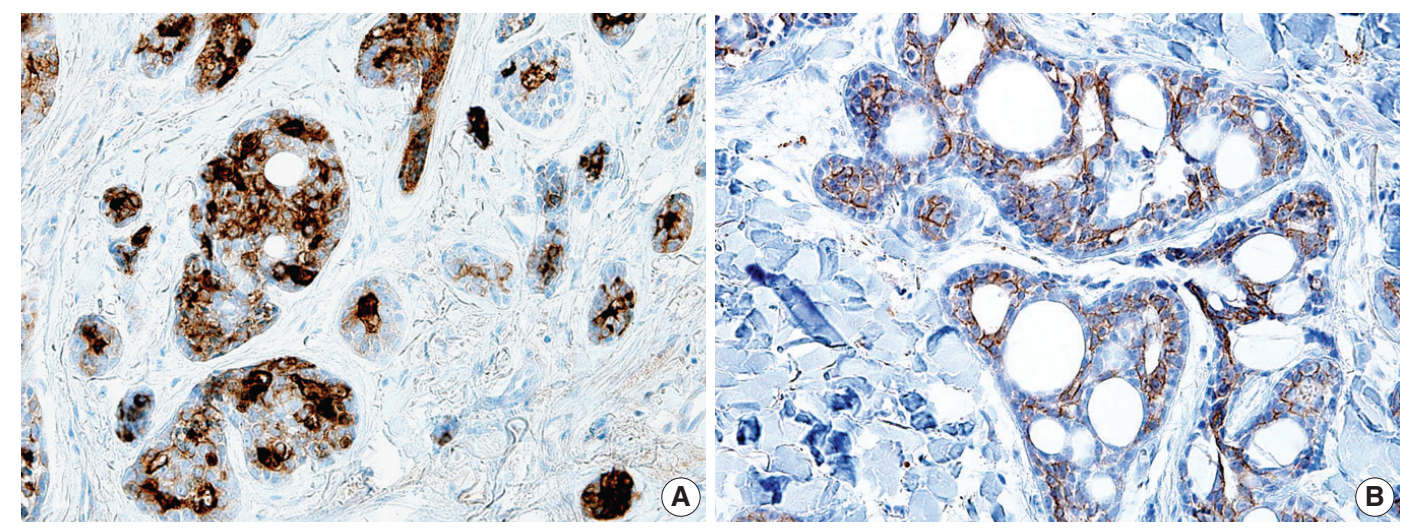

Fig. 3. Immunohistochemical staining results of primary cutaneous adenoid cystic carcinoma. Immunohistochemical staining images showing strong expression of epithelial membrane antigen (A) and KIT (B) in tumor cells. 
to lymph nodes and the lungs have been reported in five and six cases, respectively. ${ }^{3}$ In our case, there was no evidence of metastasis on chest computerized tomography scan.

Histologically, PCACC consists of basaloid cells arranged in a solid, tubular, or cribriform pattern. The tumor usually involves mid-to-reticular dermis, lying in a hyalinized fibrous stroma. It is usually not associated with the overlying epidermis and rarely invades underlying tissues. Cystic spaces are filled with mucin. The histologic findings of our case were compatible with these characteristics of PCACC.

It has been suggested that KIT (CD117) is a useful ancillary marker for ACC, distinguishing ACCs from other carcinomas with high sensitivity and specificity. ${ }^{5-8}$ In our case, EMA and KIT were strongly immunoreactive in the tumor, thus rendering the diagnosis of ACC. In contrast, basal cell carcinoma does not express EMA, and it shows palisading of the nuclei, stromal retraction, and continuity with the epidermis. These histological and immunohistochemical features were not detectable in our case, and thus we were able to rule out the possibility of basal cell carcinoma with features of adenoid cystic growth pattern.

The standard treatment of PCACC is a wide surgical excision with at least a $2 \mathrm{~cm}$ safety margin around the tumor, due to its frequent recurrence. ${ }^{10}$ In recent years, several cases of PCACC have been treated with Mohs micrographic surgery. ${ }^{9}$ The authors consider Mohs surgery to be superior to the traditional wideexcision method. However, considering the limited number of cases and follow-up period, we cannot make definitive conclusions at this point.

In summary, we described a rare adnexal tumor, a PCACC arising in the umbilicus. The PCACC is typical of its type, with features similar to other reported PCACCs. PCACCs are rare in general, regardless of their location, and this is the first reported case arising in the umbilicus. Medical caution with regards to the treatment options is required, as the recurrence rate of this tumor is not certain, and a long-term follow-up is warranted due to the possibly prolonged period before recurrence.

\section{Conflicts of Interest}

No potential conflict of interest relevant to this article was reported.

\section{REFERENCES}

1. Boggio R. Letter: adenoid cystic carcinoma of scalp. Arch Dermatol 1975; 111: 793-4.

2. Rocas D, Asvesti C, Tsega A, Katafygiotis P, Kanitakis J. Primary adenoid cystic carcinoma of the skin metastatic to the lymph nodes: immunohistochemical study of a new case and literature review. Am J Dermatopathol 2014; 36: 223-8.

3. Naylor E, Sarkar P, Perlis CS, Giri D, Gnepp DR, Robinson-Bostom L. Primary cutaneous adenoid cystic carcinoma. J Am Acad Dermatol 2008; 58: 636-41.

4. Ramakrishnan R, Chaudhry IH, Ramdial P, et al. Primary cutaneous adenoid cystic carcinoma: a clinicopathologic and immunohistochemical study of 27 cases. Am J Surg Pathol 2013; 37: 1603-11.

5. Holst VA, Marshall CE, Moskaluk CA, Frierson HF Jr. KIT protein expression and analysis of c-kit gene mutation in adenoid cystic carcinoma. Mod Pathol 1999; 12: 956-60.

6. Jeng YM, Lin CY, Hsu HC. Expression of the c-kit protein is associated with certain subtypes of salivary gland carcinoma. Cancer Lett 2000; 154: 107-11.

7. Penner CR, Folpe AL, Budnick SD. C-kit expression distinguishes salivary gland adenoid cystic carcinoma from polymorphous lowgrade adenocarcinoma. Mod Pathol 2002; 15: 687-91.

8. Mino M, Pilch BZ, Faquin WC. Expression of KIT (CD117) in neoplasms of the head and neck: an ancillary marker for adenoid cystic carcinoma. Mod Pathol 2003; 16: 1224-31.

9. Xu YG, Hinshaw M, Longley BJ, Ilyas H, Snow SN. Cutaneous adenoid cystic carcinoma with perineural invasion treated by mohs micrographic surgery-a case report with literature review. J Oncol 2010; 2010: 469049.

10. van der Kwast TH, Vuzevski VD, Ramaekers F, Bousema MT, Van Joost T. Primary cutaneous adenoid cystic carcinoma: case report, immunohistochemistry, and review of the literature. Br J Dermatol 1988; 118: 567-77. 